
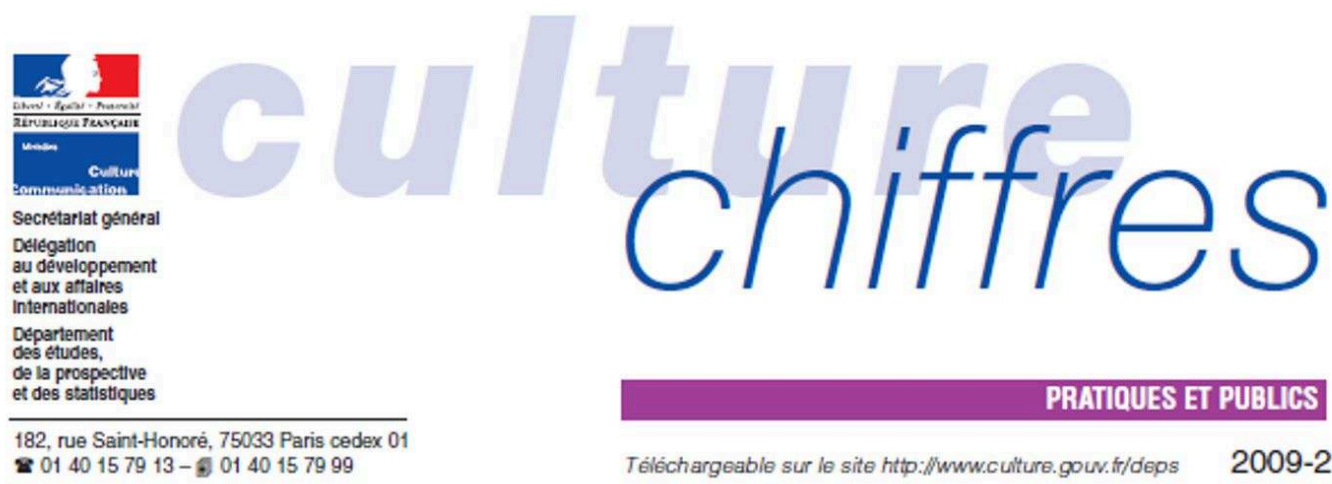

\title{
Les dépenses de consommation des ménages en biens et services culturels et télécommunications
}

\author{
Chantal Lacroix ${ }^{*}$
}

En 2007, les dépenses de consommation des ménages en France (métropole et départements d'outre-mer) dans le domaine des biens et services culturels et des télécommunications ont représenté $6,8 \%$ de la consommation totale des ménages, soit 1047 milliards d'euros tous postes confondus ou 38300 euros par ménage ${ }^{1}$.

Depuis 1960, la structure des différents postes de consommation des ménages a fortement évolué : tandis que les dépenses liées à l'alimentation, l'alcool, le tabac et l'habillement sont celles dont la part a le plus diminué, à l'inverse, les transports, le logement, les communications et les loisirs ont augmenté plus vite que la moyenne, mais à des rythmes différents pour chacun de ces postes ${ }^{2}$.

Plus de $\mathbf{7 0}$ milliards d'euros de dépenses des ménages en biens et services culturels et télécommunications en 2007

Les 70,7 milliards d'euros de dépenses des ménages en biens et services culturels et télécommunications en 2007 représentent une dépense moyenne de 2585 euros par ménage (tableau 1). Ces dépenses se répartissent en deux groupes principaux :
- plus d'un tiers est consacré aux biens et services culturels (hors matériels). Elles s'élèvent à 25,9 milliards d'euros, soit $2,5 \%$ de la consommation totale des ménages. Ces dépenses ont augmenté de $23,3 \%$ de 2000 à 2007 , mais cette augmentation demeure inférieure à celle de la consommation totale des ménages, qui est de $33,6 \%$ sur la même période. Elles représentent 25,9 milliards d'euros, soit $2,5 \%$ de la consommation totale des ménages ;

- des dépenses liées à l'utilisation des matériels ainsi qu'aux télécommunications qui sont croissantes et représentent 28,4 milliards d'euros, soit $2,7 \%$ de la consommation totale des ménages. L'essentiel de ces dépenses - 24,9 milliards d'euros - est consacré aux dépenses de téléphone et d'accès à l'internet (soit $2,4 \%$ des dépenses totales des ménages), tandis que les dépenses liées à l'utilisation des biens et services s'élèvent à 3,5 milliards d'euros. Pour leur part, les dépenses en achats de matériels, qui représentent près d'un quart des dépenses culturelles, paraissent stables (16,4 milliards d'euros), mais sont en réalité caractérisées, ces dernières années, par une hausse importante des achats, compensée par la baisse générale du prix des équipements.

\footnotetext{
* Chargée d'études au nerps.

1. En 2007 , le nombre de ménages en France métropolitaine et territoires d'outre-mer est estimé à 27,4 millions. Insee, Consommation des ménages, tableaux détaillés, les comptes de la nation en 2007 - Base 2000, http $/ /$ wwwinsee.fr/fr/themes/comptes-nationaux/souschapitre.asp Xdd=73

2. Jérôme Accrsno, * 1979-2006: les structures de consommation évoluent et les écarts entre groupes sociaux se déplacent s, France portrait social, édition 2007 , Insee, novembre 2007.
} 


\section{Les dépenses de consommation des ménages en biens et services culturels et télécommunications}

\section{Chantal Lacroix}

Éditeur : Département des études, de la prospective et des statistiques

Lieu d'édition : Paris

Année d'édition : 2009

Date de mise en ligne : 21 septembre 2015

Collection : Culture chiffres

ISBN électronique : 9782111398436

\section{Gbooks}

http://books.openedition.org

\section{Édition imprimée}

Date de publication : 1 mars 2009

Nombre de pages : 8

\section{Référence électronique}

LACROIX, Chantal. Les dépenses de consommation des ménages en biens et services culturels et télécommunications. Nouvelle édition [en ligne]. Paris : Département des études, de la prospective et des statistiques, 2009 (généré le 25 avril 2021). Disponible sur Internet : <http:// books.openedition.org/deps/595>. ISBN : 9782111398436. 

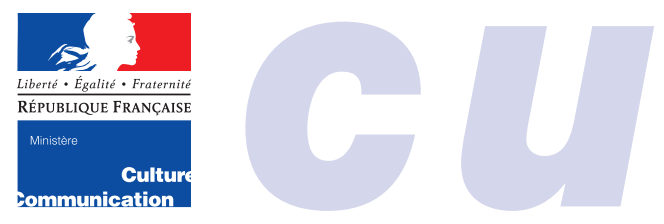

Secrétariat général

Délégation

au développement

et aux affaires

internationales

Département

des études,

de la prospective

et des statistiques

\section{Les dépenses de consommation des ménages en biens et services culturels et télécommunications}

\section{Chantal Lacroix*}

En 2007, les dépenses de consommation des ménages en France (métropole et départements d'outre-mer) dans le domaine des biens et services culturels et des télécommunications ont représenté $6,8 \%$ de la consommation totale des ménages, soit 1047 milliards d'euros tous postes confondus ou 38300 euros par ménage ${ }^{1}$.

Depuis 1960, la structure des différents postes de consommation des ménages a fortement évolué : tandis que les dépenses liées à l'alimentation, l'alcool, le tabac et l'habillement sont celles dont la part a le plus diminué, à l'inverse, les transports, le logement, les communications et les loisirs ont augmenté plus vite que la moyenne, mais à des rythmes différents pour chacun de ces postes ${ }^{2}$.

\section{Plus de 70 milliards d'euros de dépenses des ménages en biens et services culturels et télécommunications en 2007}

Les 70,7 milliards d'euros de dépenses des ménages en biens et services culturels et télécommunications en 2007 représentent une dépense moyenne de 2585 euros par ménage (tableau 1). Ces dépenses se répartissent en deux groupes principaux :
- plus d'un tiers est consacré aux biens et services culturels (hors matériels). Elles s'élèvent à 25,9 milliards d'euros, soit $2,5 \%$ de la consommation totale des ménages. Ces dépenses ont augmenté de 23,3\% de 2000 à 2007, mais cette augmentation demeure inférieure à celle de la consommation totale des ménages, qui est de $33,6 \%$ sur la même période. Elles représentent 25,9 milliards d'euros, soit $2,5 \%$ de la consommation totale des ménages ;

- des dépenses liées à l'utilisation des matériels ainsi qu'aux télécommunications qui sont croissantes et représentent 28,4 milliards d'euros, soit $2,7 \%$ de la consommation totale des ménages. L'essentiel de ces dépenses - 24,9 milliards d'euros - est consacré aux dépenses de téléphone et d'accès à l'internet (soit 2,4\% des dépenses totales des ménages), tandis que les dépenses liées à l'utilisation des biens et services s'élèvent à 3,5 milliards d'euros. Pour leur part, les dépenses en achats de matériels, qui représentent près d'un quart des dépenses culturelles, paraissent stables (16,4 milliards d'euros), mais sont en réalité caractérisées, ces dernières années, par une hausse importante des achats, compensée par la baisse générale du prix des équipements.

\footnotetext{
* Chargée d'études au DEPS.

1. En 2007, le nombre de ménages en France métropolitaine et territoires d'outre-mer est estimé à 27,4 millions. Insee, Consommation des ménages, tableaux détaillés, les comptes de la nation en 2007 - Base 2000, http://www.insee.fr/fr/themes/comptes-nationaux/souschapitre.asp?id=73

2. Jérôme ACCARDO, « 1979-2006 : les structures de consommation évoluent et les écarts entre groupes sociaux se déplacent », France portrait social, édition 2007, Insee, novembre 2007.
} 


\section{Postes de la nomenclature des dépenses de consommation des ménages retenus pour décrire la consommation en biens et services culturels et télécommunications}

Biens et services culturels hors matériels

- Livres

- Journaux

- Revues et périodiques

- Disques

- Vidéos

- Logiciels, y compris jeux vidéo

- Téléchargement

- Redevance radio

- Redevance et abonnements TV

- Cinéma

- Spectacles (activités marchandes)
Matériels

- Postes de radio

- Téléviseurs

- Lecteurs et/ou enregistreurs du son et de l'image, y compris appareils photo numériques et caméscopes

- Appareils photo argentiques et instruments d'optique

- Ordinateurs et équipements associés

- Instruments de musique
Biens et services liés à ces matériels

- Pellicules, papier photo

- Supports vierges audio, vidéo, informatique

- Réparation de matériel électronique grand public

- Entretien et réparation de machines de bureau et de matériel informatique

- Activités photographiques

\section{Télécommunications}

- Ce poste comprend les abonnements téléphoniques et à l'internet

La nomenclature par produit (en correspondance avec la nomenclature d'activités française Naf) est la plus détaillée pour l'analyse des dépenses de consommation des ménages dans la comptabilité nationale. Cependant, certains postes du domaine culturel sont identifiables, tandis que d'autres sont regroupés dans des postes plus larges, sans qu'il soit possible de les dissocier de ce qui n'entre pas dans le champ culturel. Ainsi les jeux vidéo sont-ils assimilés aux logiciels, le matériel de dessin associé à la papeterie ou encore les activités culturelles non marchandes comme les visites patrimoniales du secteur public, intégrées à l'ensemble des activités récréatives, culturelles et sportives non marchandes. D'autre part, certaines dépenses des ménages, comme les dépenses en œuvres d'art et antiquités ${ }^{1}$ ou en visites patrimoniales du secteur privé, sont absentes des données de la comptabilité nationale.

Enfin, des choix ont dû être faits parmi les postes existants pour refléter au mieux les dépenses de consommation des ménages dans le domaine culturel. Ainsi, les matériels tels que les téléviseurs, qui n'ont pas de contenu culturel en soi mais dont l'acquisition est une condition nécessaire pour accéder à certains biens et services culturels comme les programmes télévisés, ont été retenus. Les biens et services liés à l'utilisation de ces matériels (à l'exception de l'accès à l'internet qui est compris dans le poste Télécommunications) font également partie du champ (supports vierges d'enregistrement audio et vidéo par exemple).

Le développement de la dématérialisation des supports des œuvres et leur accès par l'intermédiaire d'internet impliquent également de prendre en compte les dépenses de télécommunications des ménages. Bien que ces derniers postes, matériels et télécommunications, puissent être a priori considérés comme connexes au champ des dépenses culturelles au sens strict, ils ont toute leur place dans cette analyse, compte tenu des arbitrages que font les ménages entre ces différentes dépenses.

1. Pour les œuvres d'art et les antiquités, leur absence est due à la méthodologie utilisée pour la construction des comptes nationaux, qui les exclut. « La consommation des ménages, sources et méthodes ", Insee méthodes, version du 6 juillet 2007, p. 2. http://www.insee.fr/fr/methodes/sources/pdf/consommation_menages.pdf

\section{Le renversement de la structure des dépenses culturelles et de télécommunications}

En cinquante ans, les poids relatifs des quatre types de dépenses ont beaucoup évolué, opérant un renversement entre la part des dépenses en biens et services culturels et celle des autres types de dépenses de consommation culturelles des ménages (graphique 1). De $58 \%$ en 1959, cette dernière est passée à $37 \%$ en 2007 , tandis que la part cumulée des dépenses de matériels, services liés à ces matériels et dépenses de télécommunications est passée de $42 \%$ à $63 \%$.

Le développement des dépenses de télécommunications dont le poids relatif est passé de $8 \%$ à $35 \%$ explique l'essentiel de ce changement, alors que la part des matériels est stable ( $23 \%$ en 1959 comme en 2007) et que celle des biens et services liés à l'utilisation des matériels a diminué, passant de $11 \%$ en 1959 à $5 \%$ en 2007.

La diffusion du téléphone au cours des années 1970 et 1980 est telle qu'en 1983, les montants des dépenses de télécommunications dépassent pour la première fois celles pour les matériels, bien avant que les dépenses liées à l'utilisation de l'internet par les ménages n'apparaissent dans leurs dépenses. On notera d'ailleurs que le taux d'équipement des ménages était de $13 \%$ en 1960 pour les téléviseurs et de moins de $10 \%$ en 1963 pour le téléphone. Le taux de $90 \%$ a été atteint en 1981 pour les téléviseurs puis en 1983 pour le téléphone. Le développement de l'équipement des ménages en microordinateur ne commence que vers la fin des années 1990, 15 \% des ménages en possèdent en 1995, ils sont $27 \%$ en 2000 et $62 \%$ au début de l'année $2008^{3}$.

3. Martine DAYAn et Raymond HeItzMann, Tableau de bord des Tic et du commerce électronique, entreprises-ménages, ministère de l'Économie, de l'Industrie et de l'Emploi, Service des études et des statistiques industrielles, décembre 2008. 
Tableau 1 - Dépenses de consommation des ménages en biens et services culturels et connexes

\begin{tabular}{|c|c|c|c|c|c|c|c|c|c|}
\hline & \multirow{2}{*}{$\begin{array}{c}\text { Millions } \\
\text { d'euros }\end{array}$} & \multicolumn{3}{|c|}{$\begin{array}{l}\text { Évolution } \\
\text { en volume* }\end{array}$} & \multicolumn{3}{|c|}{$\begin{array}{l}\text { Évolution } \\
\text { des prix }\end{array}$} & \multicolumn{2}{|c|}{$\begin{array}{c}\text { Poids dans } \\
\text { la valeur de la } \\
\text { consommation } \\
\text { (en \%) }\end{array}$} \\
\hline & & 2005 & 2006 & 2007 & 2005 & 2006 & 2007 & 1997 & 2007 \\
\hline Biens et services culturels & 25923 & & & & & & & 2,62 & 2,48 \\
\hline Redevance et abonnements TV & 5433 & 1,3 & 1,1 & 1,8 & 2,8 & 2,5 & 2,7 & 0,47 & 0,52 \\
\hline Revues et périodiques & 4593 & $-1,0$ & 1,3 & $-1,2$ & 2,1 & 1,0 & 1,7 & 0,55 & 0,44 \\
\hline Spectacles (activités marchandes) & 4336 & 5,3 & 6,2 & 6,5 & 1,2 & 2,5 & 3,8 & 0,23 & 0,41 \\
\hline Livres & 3627 & $-1,1$ & $-1,1$ & 1,2 & 0,7 & 1,1 & 1,1 & 0,38 & 0,35 \\
\hline Journaux & 2439 & $-1,2$ & $-6,2$ & $-1,4$ & 1,9 & 1,2 & 2,8 & 0,34 & 0,23 \\
\hline Vidéos & 1684 & 13,7 & $-2,8$ & $-5,1$ & $-19,9$ & $-4,4$ & $-5,9$ & 0,11 & 0,16 \\
\hline Cinéma & 1144 & $-11,6$ & 6,7 & $-6,9$ & 2,5 & 2,6 & 2,3 & 0,12 & 0,11 \\
\hline Disques & 955 & $-2,9$ & $-10,4$ & $-16,1$ & $-1,8$ & $-2,1$ & $-4,8$ & 0,26 & 0,09 \\
\hline Téléchargement & 610 & 6,9 & 11,5 & 10,2 & 1,5 & 1,1 & 0,5 & 0,03 & 0,06 \\
\hline Redevance radio & 604 & 1,6 & $-0,9$ & $-1,5$ & 2,6 & 2,6 & 2,5 & 0,06 & 0,06 \\
\hline Logiciels, y compris jeux vidéo & 498 & $-2,6$ & 3,2 & 9,8 & $-4,1$ & $-3,5$ & $-3,7$ & 0,06 & 0,05 \\
\hline Matériels & 16427 & & & & & & & 1,21 & 1,57 \\
\hline Ordinateurs et équipements associés & 6398 & 24,2 & 19,3 & 18,6 & $-15,2$ & $-12,7$ & $-13,3$ & 0,42 & 0,61 \\
\hline Téléviseurs & 4708 & 31,7 & 49,9 & 49,6 & $-11,8$ & $-14,0$ & $-22,2$ & 0,24 & 0,45 \\
\hline Lecteurs, enregistreurs son et image ${ }^{\star *}$ & 4025 & 31,5 & 15,9 & 11,4 & $-14,1$ & $-10,9$ & $-13,0$ & 0,23 & 0,38 \\
\hline Postes de radio & 843 & $-7,4$ & $-0,8$ & 4,0 & $-8,8$ & $-9,1$ & $-10,3$ & 0,20 & 0,08 \\
\hline Appareils photo argentiques et instruments d'optiqu & que 230 & $-15,7$ & $-25,5$ & 0,4 & $-2,9$ & $-6,5$ & 0,0 & 0,10 & 0,02 \\
\hline Instruments de musique & 223 & 4,0 & 9,7 & 1,3 & $-0,5$ & $-0,9$ & $-1,8$ & 0,03 & 0,02 \\
\hline Biens et services liés aux matériels & 3518 & & & & & & & 0,56 & 0,34 \\
\hline Activités photographiques & 1461 & $-7,6$ & $-7,6$ & 0,1 & 0,8 & $-0,3$ & 0,5 & 0,25 & 0,14 \\
\hline Réparation de matériel électronique grand public & 959 & 1,1 & 3,2 & 3,8 & 2,3 & 2,2 & 2,8 & 0,11 & 0,09 \\
\hline Supports vierges audio, vidéo, informatique & 529 & 4,7 & 1,4 & 6,4 & $-2,8$ & $-9,2$ & $-14,3$ & 0,07 & 0,05 \\
\hline Entretien et réparation de matériel informatique & 380 & 0,5 & $-1,1$ & 1,6 & 0,8 & 1,6 & 0,5 & 0,04 & 0,04 \\
\hline Pellicules, papier photo & 189 & $-37,0$ & $-34,3$ & $-15,7$ & $-0,3$ & $-1,3$ & 0,5 & 0,09 & 0,02 \\
\hline Télécommunications & 24873 & & & & & & & 1,53 & 2,37 \\
\hline Télécommunications (téléphone et accès internet) & 24873 & 5,7 & 9,0 & 5,6 & 0,2 & $-6,2$ & $-1,1$ & 1,53 & 2,37 \\
\hline $\begin{array}{l}\text { Total biens et services culturels } \\
\text { et télécommunications }\end{array}$ & 70741 & & & & & & & 5,93 & 6,75 \\
\hline Dépenses totales des ménages & 1047356 & 2,6 & 2,3 & 2,5 & 1,8 & 2,2 & 2,0 & 100,00 & 100,00 \\
\hline
\end{tabular}

Source : Insee, comptes nationaux/DEPS, ministère de la Culture et de la Communication, 2009.

Graphique 1 - Évolution des parts relatives des quatre grands postes de dépenses des ménages en biens et services culturels et télécommunications au cours des cinquante dernières années (en valeur)

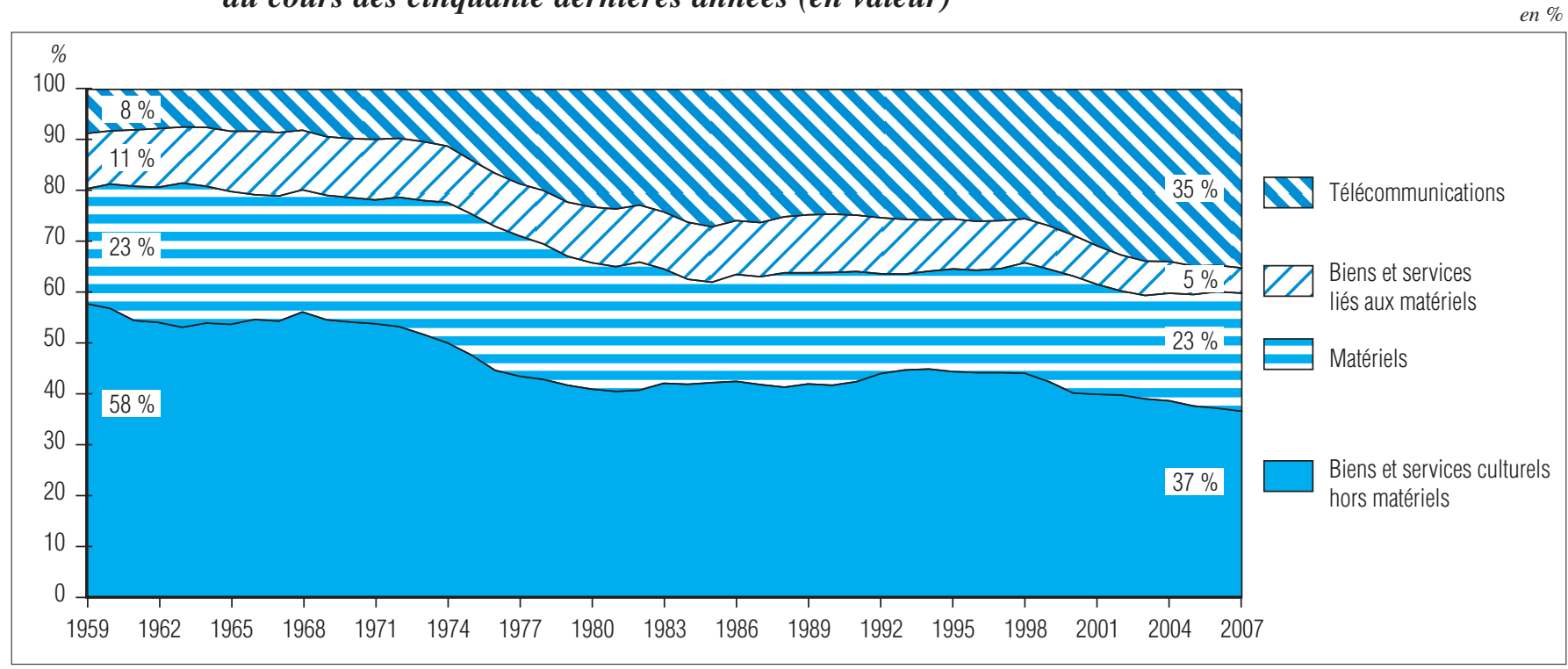

Source : Insee, comptes nationaux/DEPS, ministère de la Culture et de la Communication, 2009 


\section{Des disparités dans les évolutions en prix et volume depuis 2000}

Les tendances de consommation des ménages peuvent s'expliquer pour une large part en fonction de l'évolution des prix et des volumes. Peu de biens et services liés à la culture connaissent une évolution des prix supérieure à celle des dépenses totales des ménages: seuls les prix des spectacles marchands et du cinéma dépassent régulièrement celleci. Autrement dit, pour un ménage dont les revenus évoluent suivant l'indice des prix, la consommation en spectacles marchands et cinéma coûte de plus en plus cher, tandis que les autres biens et services culturels enregistrent une croissance des prix inférieure ou équivalente à celle des prix, en particulier les biens et services liés aux matériels, et les télécommunications ${ }^{4}$.

Selon cette logique, on peut ordonner en cinq groupes les biens et services culturels et télécommunications à partir de leurs évolutions en prix et volume depuis 2000 (graphique 2).

\section{Matériels de premier équipement ou de renouvellement : une baisse des prix corrélée à une augmentation en volume des produits consommés}

Les biens et services culturels dont les dépenses en volume ont le plus augmenté depuis 2000 sont les téléviseurs, ordinateurs, lecteurs enregistreurs audio et vidéo dont les appareils photo numériques (graphique 2, zone 1). Les prix de ces appareils ont parallèlement fortement diminué au cours de cette même période. Un double mouvement qui s'explique par les constantes évolutions technologiques de ces appareils qui induisent des dépenses de renouvellement ou de premier équipement.

Le marché des téléviseurs est un exemple du renouvellement d'équipement, avec le remplacement progressif des appareils cathodiques par des écrans plats. Depuis 2000, la dépense en téléviseurs des ménages, de 2,1 milliards d'euros, a plus que doublé en valeur, passant à 4,7 milliards d'euros en

\section{Graphique 2 - Évolution de 2000 à 2007 des indices de prix et de volume des biens et services culturels et télécommunications}

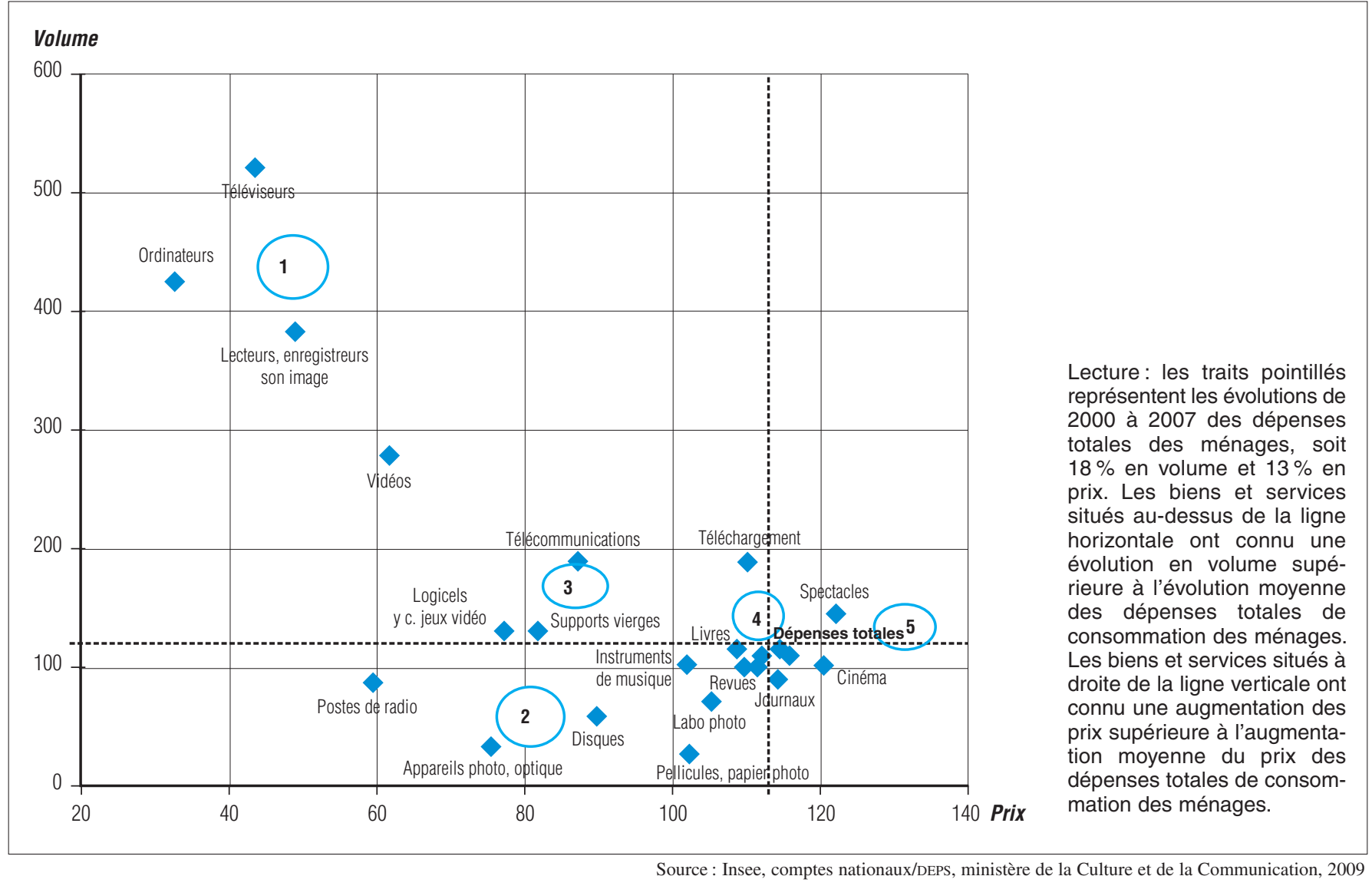

4. Voir Régis ARthaud, «La consommation des ménages en Tic depuis 45 ans, un renouvellement permanent », Insee Première, $\mathrm{n}^{\circ} 1101$, septembre 2006, http://www.insee.fr/fr/themes/document.asp?ref_id=ip1101\&reg_id=0 
2007. Les prix ayant été divisés par plus de deux $(-56,5 \%)$, les volumes ont été multipliés par cinq. Le marché des micro-ordinateurs conjugue encore premier équipement et renouvellement pour des appareils plus performants. La dépense des ménages pour ce bien a augmenté de $30 \%$ sur cette période, les prix ont baissé de $70 \%$ et les volumes ont été multipliés par quatre.

\section{Matériels affectés par la révolution numérique : baisse des prix et des volumes}

Ce deuxième groupe comprend tout ce qui est lié à la photo argentique, mais aussi les postes de radio et les disques. Les vidéos pourraient être rattachées au premier groupe (les dépenses en volume ont été multipliées par trois de 2000 à 2005 avec le développement du DVD) mais la tendance récente de la baisse du volume des ventes (- $8 \%$ de 2005 à 2007) les rapproche plutôt du deuxième groupe.

Le remplacement de la photo argentique par le numérique entraîne une chute du volume des ventes des appareils (-65,5\% de 2000 à 2007) malgré une baisse des prix de $25 \%$, ainsi que des pellicules et des services liés comme les développements.

\section{Biens et services liés aux nouvelles technologies : baisse des prix et croissance modérée en volume}

Un troisième groupe se caractérise par une baisse des prix conjuguée à une croissance en volume mais moindre que celles du groupe un. Il rassemble les biens et services liés aux nouvelles technologies : les abonnements téléphoniques et à l'internet, les dépenses de téléchargement, les logiciels dont les jeux vidéo et les supports vierges audio, vidéo et informatique.

\section{Biens culturels peu concernés par les évolutions technologiques : évolutions en prix et en volume semblables aux dépenses totales des ménages}

Le quatrième groupe correspond aux biens et services dont les évolutions en prix et en volume sont proches des évolutions de l'ensemble des dépenses de consommation des ménages. Il comprend les biens culturels qui n'ont pas connu d'évolutions technologiques majeures (les livres, les journaux, revues et périodiques) ainsi que des services comme la réparation et l'entretien des matériels, la redevance et les abonnements TV.

\section{Cinéma et spectacle : hausse des prix supérieure aux dépenses totales}

Le dernier groupe comprend deux types de services culturels, le cinéma et les spectacles, qui ont connu une évolution des prix supérieure à celle de l'ensemble de la consommation, mais qui divergent pour les évolutions en volume. Tandis que l'indice de volume des spectacles augmente significativement $(+46,5 \%)$ celui du cinéma reste stable et est ainsi inférieur à la progression de celui de l'ensemble des dépenses des ménages $(+18 \%)$.

\section{Les dépenses consacrées} au livre et à la presse

En 2007, les dépenses de consommation des ménages pour l'écrit s'élèvent à 10,7 milliards d'euros dont les deux tiers pour la presse. Depuis 2000, ces dépenses ont augmenté de $8,9 \%$ en valeur contre $33,6 \%$ pour l'ensemble de la consommation des ménages. Toutefois, ce sont les dépenses pour le livre qui ont le plus augmenté $(24,8 \%)$, suivies par celles des revues et périodiques $(9,7 \%)$. Quant aux dépenses pour les journaux, elles ont stagné. Depuis 2003, la progression des dépenses en valeur de ces trois produits reste inférieure à celle des dépenses totales des ménages (graphique 3).

\section{Graphique 3 - Évolution des dépenses consacrées à l'écrit}

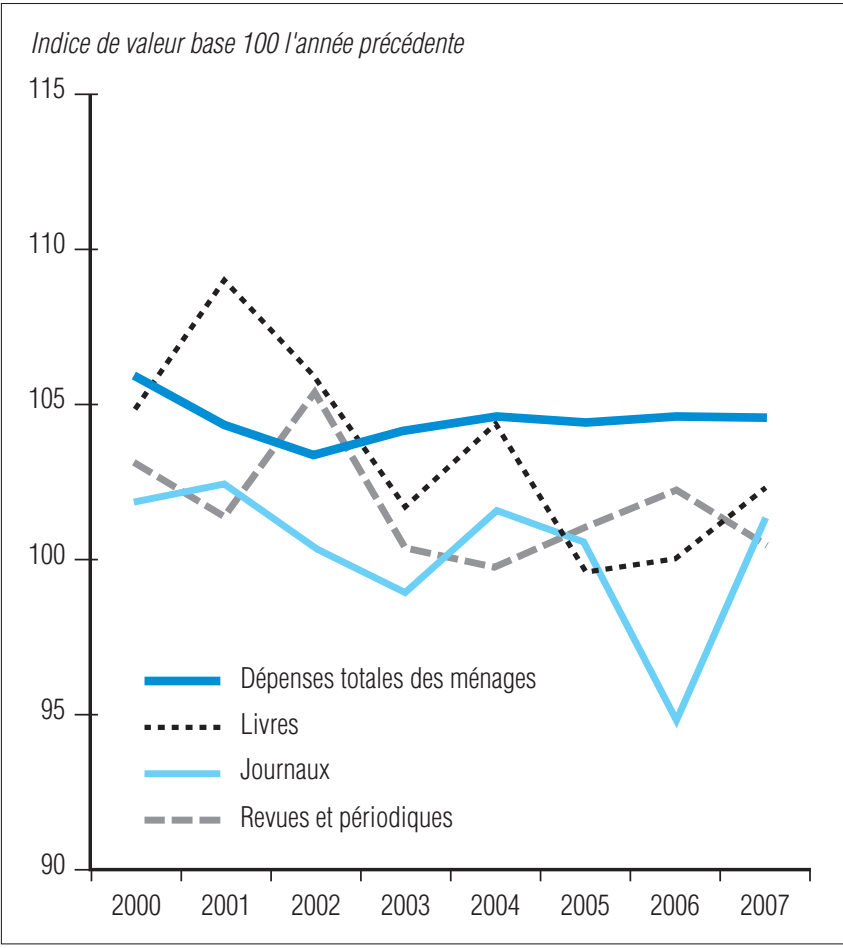

Source : Insee, comptes nationaux/DEPS, ministère de la Culture et de la Communication, 2009 
La diffusion de la presse payante est passée de 5,2 milliards d'exemplaires en 2000 à 4,6 milliards en 2006, une baisse qui affecte toutes les catégories de la presse payante ${ }^{5}$.

\section{Les disques et les vidéos : transition vers la dématérialisation}

En 2007, les ménages ont consacré 1,7 milliard d'euros aux achats de vidéos (non compris les dépenses de location) et près d'un milliard aux achats de disques (graphique 4). Ces deux biens ont connu de fortes évolutions technologiques : passage du disque vinyle et des cassettes audio au disque compact (CD) lancé sur le marché en 1992 ; apparition des premiers magnétoscopes à destination du grand public en 1974, qui a entraîné la diffusion sur le marché des vidéocassettes au format vHS dans les années 1980. Le passage au DVD s'effectue à partir de $1997^{6}$, tandis que le nouveau format Blu-ray tarde à s'implanter. C'est en 2002 que le nombre de DVD vendus par les éditeurs vidéo a été pour la première fois supérieur au nombre de cassettes vHs vendues. L'apparition du numérique conjuguée à une baisse des prix (graphique 5) entraîne une forte baisse des dépenses pour les disques depuis 2002 (le montant des dépenses des ménages consacrées à ce support a été divisé par deux entre 2002

Graphique 4 - Évolution des dépenses des ménages en disques, vidéos et téléchargements

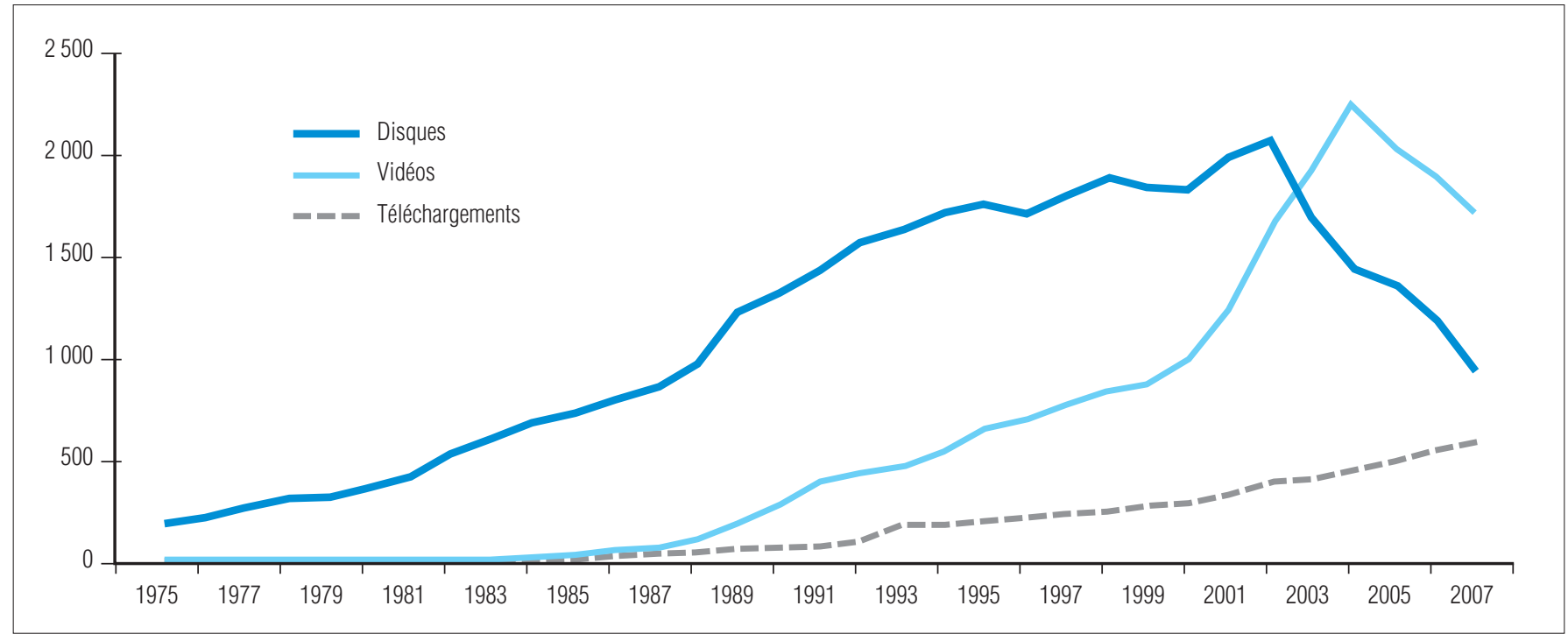

Source : Insee, comptes nationaux/DEPS, ministère de la Culture et de la Communication, 2009

Graphique 5 - Évolution des indices de prix des disques et des vidéos

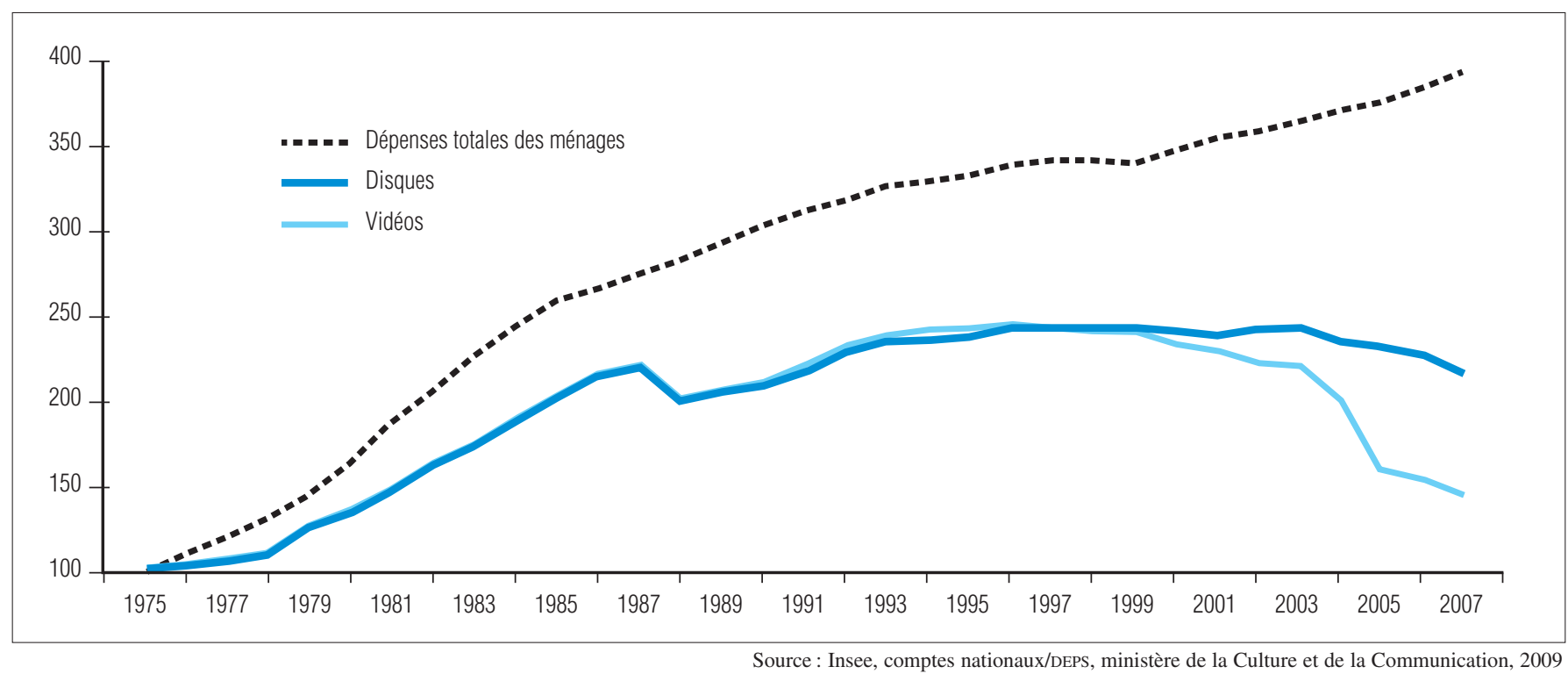

5. Tableaux statistiques de la presse, édition 2008, résultats détaillés annuels 2006, Direction du développement des médias, http://www.ddm.gouv.fr/ IMG/pdf/TSP_2008.pdf

6. Bilan et perspectives vidéo et DVD, Syndicat de l'édition vidéo numérique, Musique info Hebdo, 7e édition, 2007. 
et 2007) et à un rythme analogue mais décalé de deux ans pour la vidéo.

Les dépenses de téléchargement, qui ne concernent pas uniquement les disques et les vidéos mais aussi d'autres types de produits culturels, ne compensent pas ces baisses de consommation. En 2007, les produits les plus achetés en téléchargement ou en consultation en ligne sont les livres, journaux, revues et matériel éducatif ( $58 \%$ des acheteurs en ligne sont concernés par cet achat), viennent ensuite les films et la musique ( $47 \%$ des acheteurs en ligne) puis les logiciels, dont les jeux vidéo (22\% des acheteurs en ligne ${ }^{7}$ ).

\section{Le cinéma et les spectacles du secteur marchand ${ }^{8}$}

Les dépenses des ménages en cinéma et spectacles s'élèvent à près de 5 milliards d'euros en 2007 , dont près de $80 \%$ pour les seuls spectacles du secteur marchand. Si le poids du cinéma dans les dépenses totales des ménages est identique en 1997 et 2007, il n'en est pas de même pour les spectacles dont le poids des dépenses a doublé au cours de la même période. Les évolutions des prix de ces deux services sur cette même période sont similaires et toujours supérieures à l'évolution des prix de l'ensemble des dépenses des ménages (tableau 2).

\section{Tableau 2 - Évolution des indices de volume et de prix pour le cinéma et les spectacles du secteur marchand}

\begin{tabular}{|c|c|c|c|c|c|c|}
\hline & 1960 & 1970 & 1980 & 1990 & 2000 & 2007 \\
\hline \multicolumn{7}{|l|}{ Indices des prix } \\
\hline Dépenses totales des ménages & 100,0 & 150,2 & 374,3 & 695,0 & 796,7 & 900,5 \\
\hline Cinéma & 100,0 & 204,8 & 578,2 & 1229,8 & 1509,2 & 1815,8 \\
\hline Spectacles & 100,0 & 175,3 & 469,8 & 801,8 & 1157,2 & 1410,5 \\
\hline \multicolumn{7}{|l|}{ Indices de volume } \\
\hline Dépenses totales des ménages & 100,0 & 170,9 & 247,1 & 313,1 & 369,6 & 436,9 \\
\hline Cinéma & 100,0 & 65,1 & 65,1 & 41,4 & 51,6 & 52,0 \\
\hline Spectacles & 100,0 & 116,6 & 191,7 & 218,0 & 406,0 & 594,8 \\
\hline
\end{tabular}

Source : Insee, comptes nationaux/DEPS, ministère de la Culture et de la Communication, 2009.

7. Martine DAYAn et Raymond HeitzMAnN, art. cité. Voir aussi Pierre BerRet, Diffusion et utilisation des Tic en France et en Europe, Paris, DEPS/ministère de la Culture et de la Communication, coll. «Culture chiffres », 2008-2, p. 6.

8. Le critère marchand retenu par la comptabilité nationale regroupe des spectacles de natures très diverses : représentations théâtrales, concerts mais aussi sons et lumières, salons et foires...

\section{Source des données sur la consommation des ménages}

La comptabilité nationale est l'une des sources pour l'étude des dépenses de consommation des ménages. Elle utilise elle-même diverses sources : enquêtes auprès des entreprises, données fiscales et douanières, estimations, etc. Elle fournit annuellement une évaluation de la consommation de l'ensemble des ménages. II s'agit d'un chiffre unique élaboré par l'Insee dans le cadre des comptes nationaux français établis selon le système européen de comptabilité et qui permet des analyses d'évolution à court et long terme*. Les dépenses de consommation totales des ménages utilisées dans cette analyse sont celles qui se limitent aux dépenses que les ménages supportent directement, elles se distinguent de la consommation finale effective des ménages qui comprend les " transferts sociaux en nature des administrations " (remboursements de sécurité sociale, aides aux logements, dépenses de la collectivité en éducation, santé, etc.). Les coefficients budgétaires se définissent comme la part de consommation en valeur relative à un bien ou un service particulier dans la consommation totale. L'Insee réalise également l'enquête Budget des familles auprès des ménages, destinée à mesurer leur consommation. Cette enquête, renouvelée tous les cinq ans environ, est plus apte à mettre en évidence les disparités internes aux populations enquêtées (disparités socioprofessionnelles, démographiques ou géographiques).

* Les données de 2007 sont provisoires et susceptibles d'être révisées en 2009 et 2010, les données de 2006 seront définitives en 2009 . Les données sont en ligne sur le site de l'Insee dans la rubrique Thèmes, « comptes nationaux-finances publiques » (http://www.insee.fr/fr/themes/comptes-nationaux/souschapitre.asp?id=73).

\section{La notion de volume}

«Du fait de la hausse des prix, la monnaie se déprécie au cours du temps. On peut corriger cet effet. On produit pour cela des évolutions en valeur (évaluées à prix courants, sans correction de l'effet de l'inflation) distinctes des évolutions en volume, en intégrant une correction éliminant l'effet de l'inflation. À cette fin, on calcule la consommation d'une année aux prix de l'année précédente. Pour chaque poste élémentaire, on rapporte le montant de la consommation en valeur à l'évolution des prix par rapport à l'année précédente. En comparant le chiffre ainsi obtenu et le montant en valeur de l'année précédente, on peut ensuite évaluer des évolutions (ou des indices) entre deux années consécutives, dans lesquelles l'effet direct des hausses et des baisses de prix est effacé. Les évolutions en volume sur plusieurs années sont ensuite calculées par chaînage, c'est-à-dire en multipliant les indices obtenus pour chacune de ces années. Pour la consommation des ménages, le calcul est effectué pour tous les postes de consommation et à tous les niveaux de détail. ”

La présente analyse dessine les contours d'un champ des dépenses pour la culture et les médias. Elle s'inscrit dans une série de travaux menés par le DEPS pour disposer d'une vision précise des dépenses et des consommations culturelles des ménages français. Voir également François ROUET, les Dépenses culture et médias des Français, à paraître dans la coll. « Culture études », DEPS/ministère de la Culture et de la Communication, $2^{\mathrm{e}}$ trimestre 2009. 


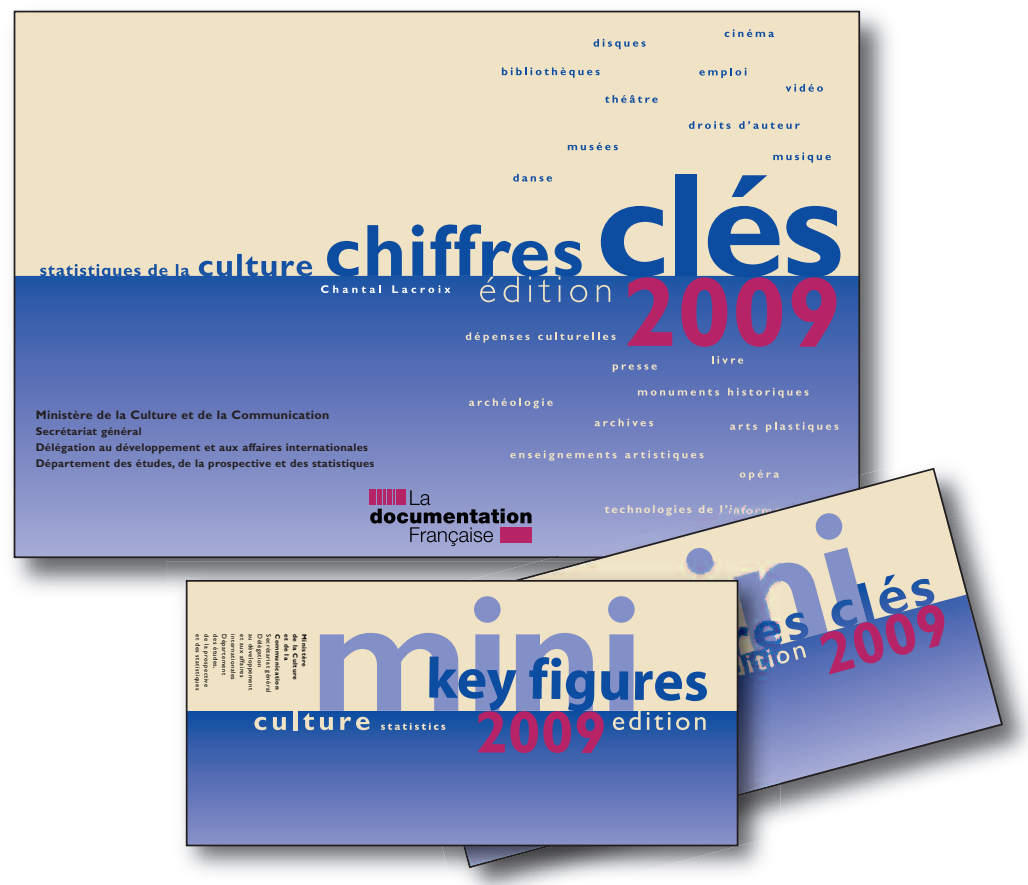

\section{Chiffres clés, statistiques de la culture}

Présentation sous forme de tableaux et graphiques des statistiques relatives à la vie culturelle en France. L'étude aborde le spectre très large du champ culturel (patrimoine, spectacle, enseignements artistiques...) et propose une approche transversale sur les grands thèmes de politique culturelle (emploi, financement, pratiques). Véritable outil de travail pour tous ceux - professionnels, responsables politiques et administratifs qui souhaitent éclairer leur action et la situer dans le contexte national, il constitue un état de la culture annuel qui fait désormais modèle. Une version abrégée, Mini chiffres clés, est également disponible en français et en anglais.

L'édition 2009 est publiée sous deux formes: imprimée (en vente à la Documentation française) et téléchargeable sur le site.

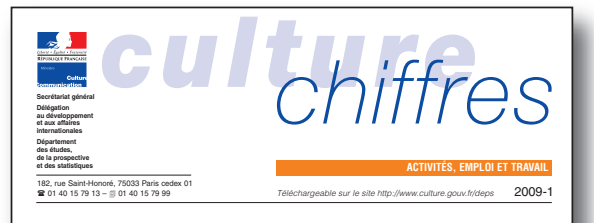

L'emploi salarié

dans le secteur de la culture

Éric Cléron, Frédérique Patureau"
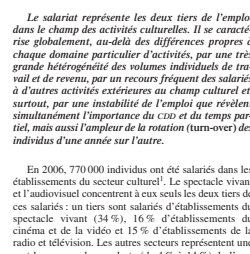

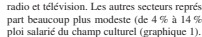

Une durée annuelle moyem

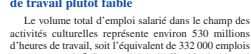

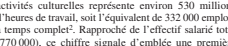

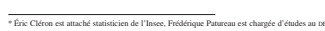

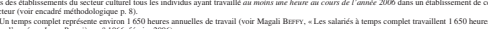

\section{L'emploi salarié}

\section{dans le secteur de la culture}

\section{Éric Cléron, Frédérique Patureau}

Le salariat représente les deux tiers de l'emploi dans le champ des activités culturelles. Il se caractérise globalement, au-delà des différences propres à chaque domaine particulier d'activités, par une très grande hétérogénéité des volumes individuels de travail et de revenu, par un recours fréquent des salariés à d'autres activités extérieures au champ culturel et, surtout, par une instabilité de l'emploi que révèlent simultanément l'importance du CDD et du temps partiel, mais aussi l'ampleur de la rotation (turn-over) des individus d'une année sur l'autre.

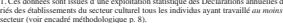

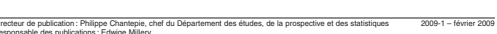

\section{Diffusion et utilisation des Tic en France et en Europe}

Pierre Berret

En quinze ans, les technologies de l'information et de la communication (Tic) sont devenues partie intégrante de la vie quotidienne des ménages et fournissent progressivement un mode d'accès élargi pour les biens et services culturels. Avec la baisse tendancielle des prix des équipements numériques (informatique, électronique grand public, abonnement au réseau internet), l'essor du haut débit, la multiplication rapide de nouvelles fonctionnalités et innovations des équipements, la diffusion et l'utilisation des Tic se sont intensifiées et démocratisées, même si ces technologies et leurs usages continuent de dépendre du niveau d'éducation, des revenus et de l'âge des usagers.

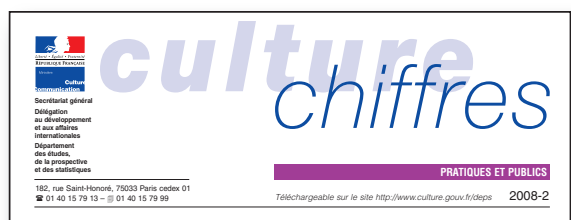

Diffusion et utilisation des TIC en France et en Europe Pierre Berret"

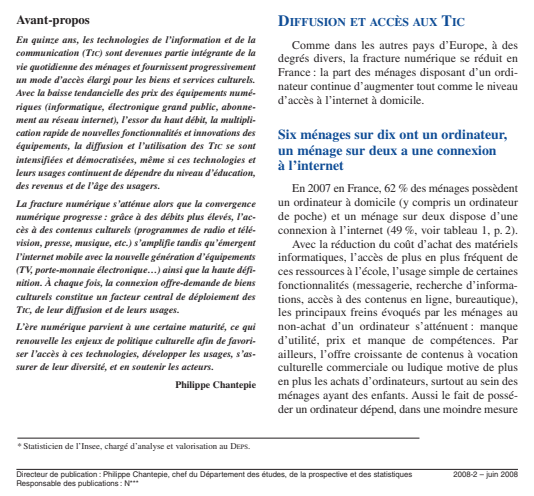

* Ces collections sont téléchargeables sur www.culture.gouv.fr/deps rubrique «publications »

Le DEPS n'assurant pas de diffusion physique de ces documents, nous vous proposons de vous informer régulièrement des parutions par message électronique. Pour ce faire, merci de bien vouloir nous communiquer votre courriel à l'adresse 